\title{
Implantable cardioverter defibrillator as a bridge to recovery in an infant with cardiac rhabdomyoma
}

\author{
Priya Chockalingam • Sally-Ann B. Clur • \\ Arthur A. M. Wilde • Irene Kuipers • Job van Woensel • \\ Nico A. Blom
}

Received: 5 July 2008 / Accepted: 4 September 2008 / Published online: 25 September 2008

(C) The Author(s) 2008. This article is published with open access at Springerlink.com

\begin{abstract}
Multiple cardiac rhabdomyomas in an infant presented with recurrent life-threatening ventricular arrhythmias refractory to medical treatment and necessitating the placement of an implantable cardioverter defibrillator (ICD). The device functioned effectively as a bridge to recovery during a 2-year follow-up period, when the tumor showed spontaneous regression, along with an almost complete resolution of the ventricular arrhythmias. We conclude that childhood cardiac rhabdomyomas causing severe drug-refractory ventricular arrhythmias can be managed by ICD therapy.
\end{abstract}

Keywords Infant · Cardiac rhabdomyoma .

Ventricular arrhythmias $\cdot$ ICD therapy

\section{Introduction}

Cardiac rhabdomyoma (CR) is the most common primary cardiac tumor in childhood, with a reported incidence of 0.02 to $0.08 \%$ in live-born infants [8]. It is predominantly a benign tumor that tends to appear between 20 and 30 weeks gestation and regresses spontaneously in the first few years

S.-A. B. Clur $\cdot$ I. Kuipers $\cdot$ N. A. Blom $(\bowtie)$

Department of Pediatric Cardiology, Academic Medical Center, G8-262,

Postbus 22660, 1100 DD Amsterdam, The Netherlands

e-mail: N.A.Blom@amc.uva.nl

P. Chockalingam $\cdot$ A. A. M. Wilde

Department of Cardiology, Academic Medical Center,

Amsterdam, The Netherlands

J. van Woensel

Pediatric Intensive Care Unit, Academic Medical Center,

Amsterdam, The Netherlands of life [6], but is strongly associated with tuberous sclerosis, which largely determines the long-term outcome [5]. The natural history of CR can be complicated by hemodynamic abnormalities, such as outflow tract obstruction, myocardial depression, or electrical abnormalities in the form of ventricular and supraventricular arrhythmias and conduction block $[1,9,10]$. We report a case of an infant with CR presenting with multiple life-threatening ventricular arrhythmias refractory to medical treatment necessitating the placement of an implantable cardioverter defibrillator (ICD).

\section{Case report}

A 40-day-old, previously healthy, female infant presented with cardiac arrest due to ventricular fibrillation (VF) at home. After successful cardiopulmonary resuscitation and defibrillation at another hospital, she was admitted to our hospital's intensive care unit. She was born prematurely at 34 weeks gestational age and with a birth weight of 2,300 g. The gravidity was uneventful, hence, an antenatal screening ultrasound was not performed in accordance with the prenatal protocol of the Netherlands at that time. There was no significant neonatal or family history.

The electrocardiogram on admission showed a sinus rhythm with normal conduction intervals and severe ST segment depression in the precordial leads (Fig. 1). The echocardiogram revealed multiple, large tumors in both ventricles, but predominantly in the left ventricle (Fig. 2a). The tumors caused no inflow or outflow tract obstruction. Since CR was suspected, a work-up for the presence of localizations of tuberous sclerosis was performed. Magnetic resonance imaging (MRI) of the brain and kidneys, and dermatological and ophthalmologic assessment showed no 


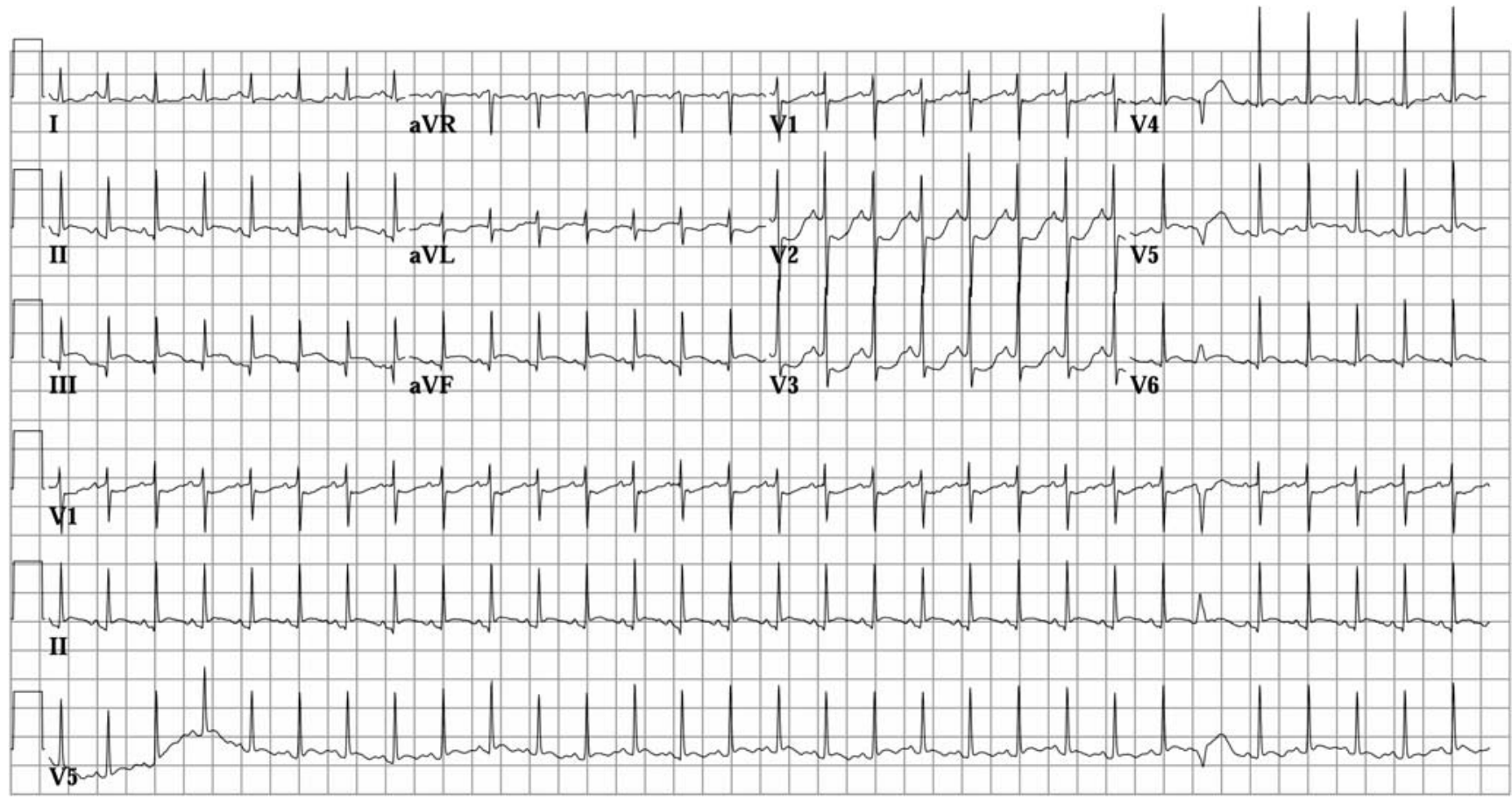

Fig. 1 Electrocardiogram on admission showing severe ST segment depression in the precordial leads
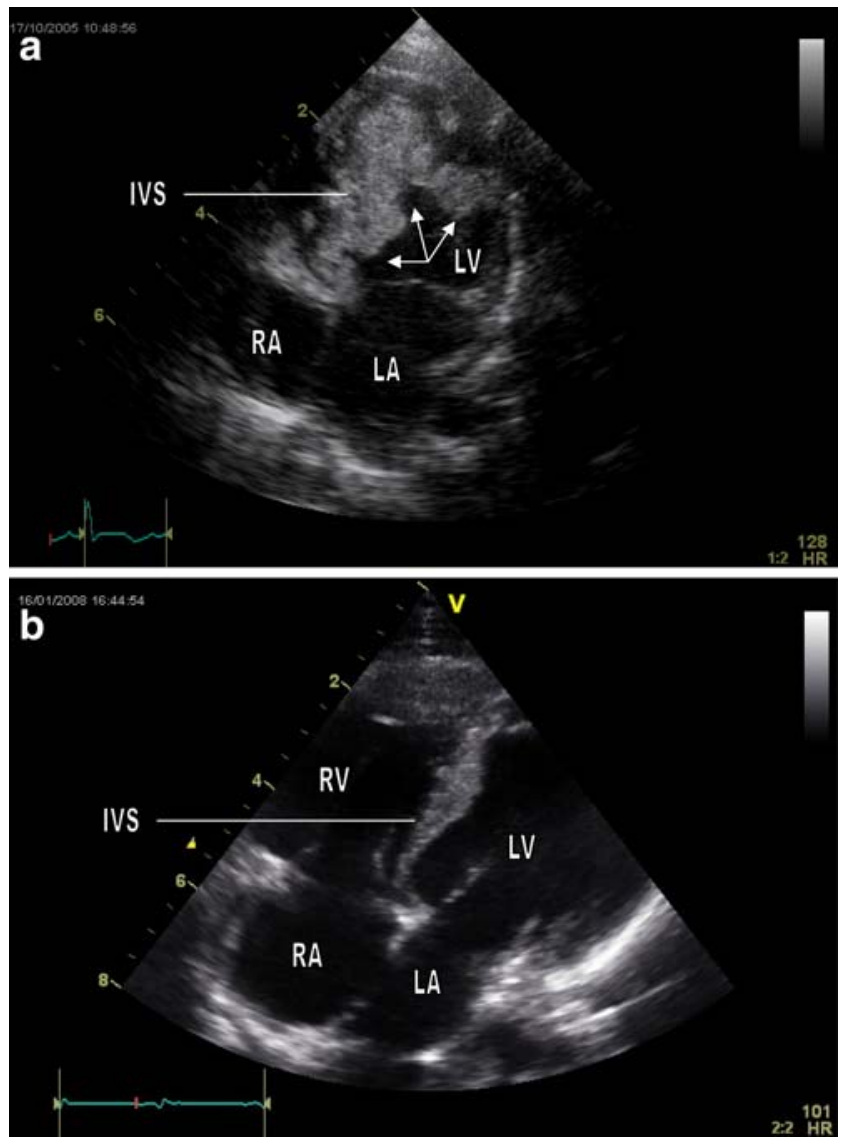

abnormalities. In addition, DNA analysis for the tuberous sclerosis (TSC2) gene was negative. Coronary angiography confirmed the echocardiographic findings and showed normal coronary artery anatomy without any compression.

The child experienced frequent episodes of very fast polymorphic ventricular tachycardia (VT), with rates of more than 300 beats per minute. There were numerous runs of nonsustained VTs lasting several seconds to minutes and, weekly, there were several episodes of fast sustained VT with loss of consciousness requiring cardiac defibrillation (Fig. 3). Ventricular arrhythmias were refractory to single-drug therapies, including beta blockers, flecainide, and amiodarone. Echocardiographic studies showed mild progression of the cardiac tumors. After 3 months in hospital, in view of refractory ventricular arrhythmias as well as tumor progression, cardiac biopsy was performed through catheterization, which confirmed the diagnosis of rhabdomyomas.

Eventually, ventricular arrhythmias could be partially suppressed with the combination of amiodarone $(10 \mathrm{mg} / \mathrm{kg})$ and flecainide $(6 \mathrm{mg} / \mathrm{kg})$. In the following months, the child's clinical condition gradually stabilized and ICD was first considered as a treatment option at this stage. Nonsustained VT episodes continued, but the occurrence of

4 Fig. 2 Echocardiography, four-chamber view. a At presentation: the arrows indicate large multiple tumors in the interventricular septum, apex, and free wall of the left ventricle. b At 2.5 years of age: the tumors have almost fully regressed. $\mathrm{LA}=$ left atrium, $\mathrm{RA}=$ right atrium, $\mathrm{LV}=$ left ventricle, $\mathrm{RV}=$ right ventricle, IVS $=$ interventricular septum 
Fig. 3 The upper panel shows the onset of ventricular tachycardia. The lower panel is the corresponding rhythm strip for the episode of ventricular tachycardia

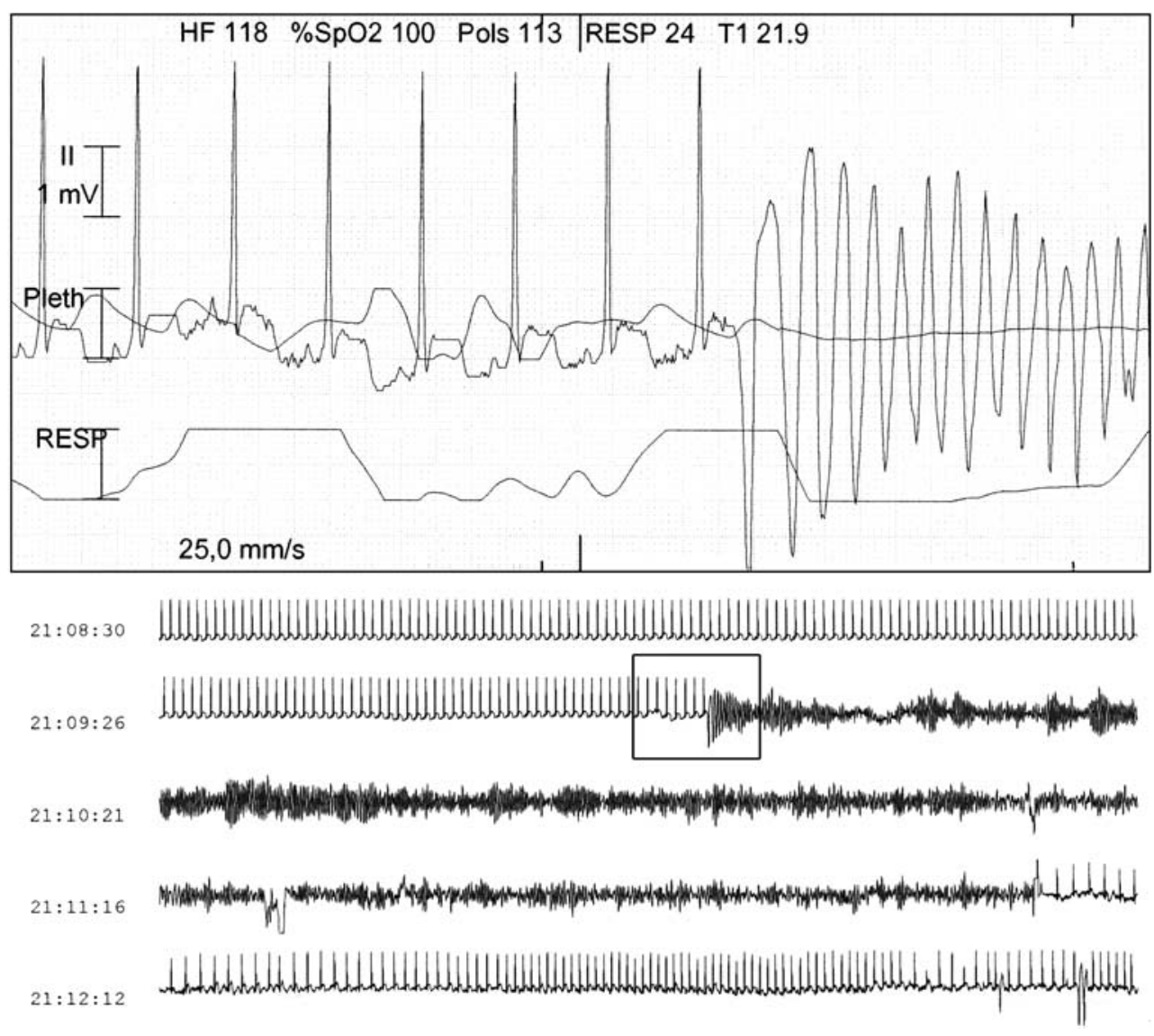

sustained VT decreased. Cardiac defibrillation, however, remained frequently necessary. At the age of 8 months, a subcutaneous ICD system was implanted. The ICD patch electrode was positioned subcutaneously in the left lateral thorax, the ventricular bipolar lead epicardially on the right ventricular apex, and the ICD generator submuscularly in the right upper abdomen. The defibrillation threshold was 10J. After a long and stormy 7-month hospital admission, the child was discharged home two weeks after ICD placement. There were no ICD shocks recorded during the period between device implant and discharge from hospital.

During 2 years of follow-up, she received three appropriate shocks for sustained polymorphic VT. ICD registrations revealed many episodes of non-sustained VTs that significantly decreased with time. The latest follow-up at the age of 2.5 years showed an almost complete disappearance of ventricular arrhythmias, correlating with near total regression of the tumors (Fig. 2b). Her neurocognitive and psychomotor development have been appropriate for age. The patient still receives daily amiodarone $8 \mathrm{mg} / \mathrm{kg}$ and flecainide $6 \mathrm{mg} / \mathrm{kg}$, and the thyroid function remains normal. The drug dosage has been planned for a gradual step-down, with a possibility of the cessation of medication in the following year. Eventually, the ICD system will be removed if the child remains free of arrhythmias without drug therapy.

\section{Discussion}

$\mathrm{CR}$ is known to regress in the postnatal period. However, in a recently published meta-analysis on antenatally diagnosed cases, it was shown that CR may have a high prenatal and neonatal mortality. The size of the cardiac tumors, fetal dysrhythmias, and hydrops were shown to be strong predictors of poor outcome [3]. CRs may be single or multiple, and are predominantly located in the ventricles and interventricular septum, and less frequently in the atria. Depending on the size and location, the tumors can also cause inflow and outflow tract obstruction. Cardiac fibroma is the next most common arrhythmia-inducing pediatric cardiac tumor. However, they are almost always single and, thus, amenable to surgical excision.

The possible electrocardiographic findings include a variety of conduction defects, prolonged PR interval, nonspecific ST-T wave changes, Wolff-Parkinson-White syndrome, and aberrant intraventricular conduction, in addition to findings of right or left ventricular hypertrophy 
and right atrial enlargement. Presenting arrhythmias in CR include supraventricular and ventricular tachycardias, as well as bradyarrhythmias.

Ventricular arrhythmias in CR can be incessant and difficult to control with antiarrhythmic drugs. The management of drug-refractory ventricular arrhythmias in infancy often requires a combination of antiarrhythmic drugs, such as flecainide with amiodarone or sotalol $[4,11]$. There are some reports of the surgical excision of solitary tumors that cause mechanical or electrical complications and, at times, surgical ablation has been sought for CR causing supraventricular tachycardia [2, 7, 14]. In the present case, single-drug therapies failed and, subsequently, different antiarrhythmic drug combinations were used. Surgical excision and ablation were not considered in this patient due to multiple widespread tumors. Ventricular arrhythmias were partially controlled with high dosages of amiodarone and flecainide; however, the risk of malignant arrhythmias and sudden cardiac death remained significant.

This is the first reported case of CR where an ICD was used as a successful approach to overcome life-threatening ventricular arrhythmias in an infant. ICDs are increasingly used in the pediatric population for both primary and secondary prevention [12]. However, pediatric ICD therapy, in addition to being limited by the small size of infants, may also be associated with complications such as infection of the device system, lead dislodgement and fracture, and a relatively higher rate of inappropriate shocks than in adults. In our patient, we used a novel technique for ICD implantation in infants as reported by Stephenson et al. [13]. The ICD generator is placed in the abdomen and a subcutaneous finger array or patch electrode is positioned in the left thorax. The ventricular sensing and pacing lead is then placed epicardially or transvenously. This technique avoids complications associated with epicardial or transvenous ICD systems, such as restrictive pericarditis, thoracotomy-related issues, and venous occlusion in young children. The subcutaneous ICD system in our patient has proved to function very effectively during a follow-up period of 2 years.

\section{Conclusion}

In conclusion, cardiac rhabdomyoma (CR) is a benign tumor that regresses during the first few years of life. However, CR can be complicated by potentially fatal ventricular arrhythmias that can be difficult to suppress with antiarrhythmic drugs. ICD therapy can act as a bridge to electrical recovery during the course of this self-limiting disease.
Open Access This article is distributed under the terms of the Creative Commons Attribution Noncommercial License which permits any noncommercial use, distribution, and reproduction in any medium, provided the original author(s) and source are credited.

\section{References}

1. Brand JM, Friedberg DZ (1992) Spontaneous regression of a primary cardiac tumor presenting as fetal tachyarrhythmias. J Perinatol 12:48-50

2. Case CL, Gillette PC, Crawford FA (1991) Cardiac rhabdomyomas causing supraventricular and lethal ventricular arrhythmias in an infant. Am Heart J 122:1484-1486. doi:10.1016/0002-8703 (91)90600-M

3. Chao AS, Chao A, Wang TH, Chang YC, Chang YL, Hsieh CC et al (2008) Outcome of antenatally diagnosed cardiac rhabdomyoma: case series and a meta-analysis. Ultrasound Obstet Gynecol 31:289-295. doi:10.1002/uog.5264

4. Fenrich AL Jr, Perry JC, Friedman RA (1995) Flecainide and amiodarone: combined therapy for refractory tachyarrhythmias in infancy. J Am Coll Cardiol 25:1195-1198. doi:10.1016/07351097(94)00513-P

5. Fesslova V, Villa L, Rizzuti T, Mastrangelo M, Mosca F (2004) Natural history and long-term outcome of cardiac rhabdomyomas detected prenatally. Prenat Diagn 24:241-248. doi:10.1002/ pd. 825

6. Giacoia GP (1992) Fetal rhabdomyoma: a prenatal echocardiographic marker of tuberous sclerosis. Am J Perinatol 9:111-114

7. Hirakubo Y, Ichihashi K, Shiraishi H, Momoi MY (2005) Ventricular tachycardia in a neonate with prenatally diagnosed cardiac tumors: a case with tuberous sclerosis. Pediatr Cardiol 26:655-657. doi:10.1007/s00246-004-0714-5

8. Isaacs H Jr (2004) Fetal and neonatal cardiac tumors. Pediatr Cardiol 25:252-273. doi:10.1007/s00246-003-0590-4

9. Mas C, Penny DJ, Menahem S (2000) Pre-excitation syndrome secondary to cardiac rhabdomyomas in tuberous sclerosis. J Paediatr Child Health 36:84-86. doi:10.1046/j.1440-1754. 2000.00443.x

10. Pipitone S, Mongiovì M, Grillo R, Gagliano S, Sperandeo V (2002) Cardiac rhabdomyoma in intrauterine life: clinical features and natural history. A case series and review of published reports. Ital Heart J 3:48-52

11. Price JF, Kertesz NJ, Snyder CS, Friedman RA, Fenrich AL (2002) Flecainide and sotalol: a new combination therapy for refractory supraventricular tachycardia in children $<1$ year of age. J Am Coll Cardiol 39:517-520. doi:10.1016/S0735-1097(01) 01773-9

12. Silka MJ, Kron J, Dunnigan A, Dick M 2nd (1993) Sudden cardiac death and the use of implantable cardioverter-defibrillators in pediatric patients. The Pediatric Electrophysiology Society. Circulation 87:800-807

13. Stephenson EA, Batra AS, Knilans TK, Gow RM, Gradaus R, Balaji $S$ et al (2006) A multicenter experience with novel implantable cardioverter defibrillator configurations in the pediatric and congenital heart disease population. J Cardiovasc Electrophysiol 17:41-46. doi:10.1111/j.1540-8167.2005.00271.x

14. Venugopalan P, Babu JS, Al-Bulushi A (2005) Right atrial rhabdomyoma acting as the substrate for Wolff-Parkinson-White syndrome in a 3-month-old infant. Acta Cardiol 60:543-545. doi:10.2143/AC.60.5.2004977 